\title{
Model Reduction of Switched Affine Systems *
}

\author{
Alessandro Vittorio Papadopoulos, Maria Prandini \\ Dipartimento di Elettronica, Informazione e Bioingegneria, Politecnico di Milano, Milano, Italy
}

\begin{abstract}
This paper addresses model reduction and extends balanced truncation to the class of switched affine systems with endogenous switching. The switched affine system is rewritten as a switched linear one with state resets that account for the affine terms. Balanced truncation can then be applied to each mode dynamics, independently. As a result, different reduced state vectors are associated with the different modes, and reset maps are here appropriately redefined so as to account and compensate for this mismatch, possibly preserving the continuity of the output. The overall behavior of the reduced switched system is determined by both the selected reduction per mode and the adopted reset maps. In this paper, we consider a stochastic setting and propose a randomized method for the selection of the reduced order. The performance of the proposed approach is illustrated through a multi-room temperature control example.
\end{abstract}

Key words: Model order reduction; Switched systems; Randomized algorithms.

\section{Introduction}

This paper addresses the design of an approximate model for a hybrid system (see e.g. [12-14,17,23,28,29]). The study of hybrid systems is typically challenging since they are characterized by intertwined continuous and discrete dynamics, [21]. Indeed, many problems that have been solved for purely discrete or purely continuous systems still lack an effective solution for hybrid systems. In particular, this is the case for the design of a reduced model.

In this paper, we focus on continuous-time Switched Affine (SA) systems with endogenous switching, and address the problem of obtaining a model that is simpler to analyze than the original system, and that is able to mimic its output behavior over a finite horizon $\mathcal{T}$. This is of interest when dealing with verification of properties that depend on the behavior of the system output over a finite horizon. Verification of properties related to the system response, like, e.g., safety and reach/avoid properties, is typically addressed in the literature through numerical methods in both the deter-

\footnotetext{
ऋ This paper was not presented at any IFAC meeting. This work was supported by the European Commission under the project UnCoVerCPS with grant number 643921. Corresponding author A. V. Papadopoulos Tel. +39-0223994028. Fax +39-02-23993412.

Email addresses: alessandro.papadopoulos@polimi.it (Alessandro Vittorio Papadopoulos), maria.prandini@polimi.it (Maria Prandini).
}

ministic, $[8,11,19,31]$, and the stochastic, $[1,2]$, settings. These methods scale exponentially with the dimension of the continuous state space component. One can then conceive a two-step procedure where an approximate abstraction with a reduced order continuous state space component is built first, and then a numerical verification method is applied to this abstraction in place of the original system.

When the input signal of the system is stochastic, the notion of approximate simulation introduced in [17] for stochastic hybrid systems [22] can be used to quantify the model performance over the output realizations. A randomized approach for assessing the performance of a given abstracted model according to this notion was proposed in [28]. The approach also extends to model design. However, no constructive procedure is given on how to select and parameterize the model class. On the contrary, in this paper we provide a constructive procedure to build an approximate model of a SA system in the form of a reduced order Switched Linear (SL) system with appropriately defined state reset maps. The SA system is first rewritten as a SL one with state reset, and then Balanced Truncation (BT) [4] is adopted for reducing the order of the linear dynamics governing the evolution of the continuous state component in each mode. State reset maps are suitably redefined accounting for the mismatch in the continuous state vectors associated with different modes. A randomized method is also proposed to determine the order of the reduced linear dynamics in each mode, while accounting for the effect of discrete transitions and state resets on the 
hybrid system evolution. The overall methodology is extended to the case when a Dwell Time (DT) is present. Note that BT is applied to switched linear systems in [27] which however deals with the case of externally induced switching. Our approach is inspired by [23] which uses BT for hybrid systems with linear dynamics and endogenous switching. The main advances with respect to [23] are: 1) the extension to the class of SA systems, 2) the introduction of novel state reset maps that provide better performance than the one adopted in [23], and of variants of these maps able to preserve continuity. Correspondingly, different initializations of the approximate model are derived based on the same logic underlying the reset maps definition, 3) the introduction of a randomized approach to select the order of the reduced linear dynamics in each mode, when the input is stochastic, and 4) the extension to the case of SA systems with DT. As a matter of fact, mode transitions and resets may strongly affect the system evolution. Indeed, the state reset map determines the new value of the continuous state after a discrete transition between modes has just occurred; while for a linear asymptotically stable system the contribution of the initial state becomes negligible in the long run, in a SA system this is generally not the case. One would in fact need to guarantee that the time between discrete transitions is sufficiently large to make the zero-input response (ZIR) vanish, which cannot be guaranteed a-priori, unless a suitable DT triggering the discrete transitions is enforced.

The choice of the order of the approximate model should then account for the influence of the state reset map on the quality of the approximation. Hence it cannot be based only on the analysis of the Hankel Singular Values (HSVs) of the linear dynamics in each mode, as suggested in [23]. The proposed randomized approach serves this purpose, since it accounts for the hybrid evolution of the candidate approximate model including mode transitions and resets. The quality of the approximation is determined also by the domains triggering the mode transitions of the SA system. Notably, redesigning the domains is quite a complex issue, [10], and it is not addressed in this paper but left for further investigation. A preliminary version of this work appeared in [24]. Additional contributions are the introduction of reset maps that preserve the output continuity, the initialization of the approximate model derived from these maps, the extension to the case of SA systems with DT, and a more thoughtful benchmark example that includes the analysis of the new reset maps and the effect of the DT. The scope of this work does not include the problem of minimal realization. To the best of our knowledge, minimal realization theory has been mainly developed for linear and bilinear switched and hybrid systems with externally induced switching, while it is still an open problem for continuous-time hybrid systems with endogenous switching [25].

\section{Switched affine systems modeling framework}

A SA system is an instance of a hybrid system, whose dynamics are characterized through a discrete state component $q_{a}$ (mode) taking values in $Q=\{1,2, \ldots, m\}$ and a continuous component $\xi_{a} \in \Xi_{a}=\mathbb{R}^{n}$ evolving according to affine dynamics that depend on the value taken by $q_{a}$. The output $y_{a} \in Y_{a}=\mathbb{R}^{p}$ of the systems is an affine function of the state and of the input $u \in U=\mathbb{R}^{m}$ that depends on $q_{a}$ as well. The continuous dynamics of a SA system within a given mode $q_{a} \in Q$ are given by

$$
\mathcal{S}_{a}:\left\{\begin{array}{l}
\dot{\xi}_{a}(t)=\mathcal{A}_{q_{a}} \xi_{a}(t)+\mathcal{B}_{q_{a}} u(t)+f_{q_{a}} \\
y_{a}(t)=\mathcal{C}_{q_{a}} \xi_{a}(t)+g_{q_{a}} .
\end{array}\right.
$$

Assumption 1 For any $i \in Q$, matrix $\mathcal{A}_{i}$ is Hurwitz, $\left(\mathcal{A}_{i}, \mathcal{B}_{i}\right)$ is controllable, and $\left(\mathcal{A}_{i}, \mathcal{C}_{i}\right)$ is observable.

As for the discrete state evolution, a collection of polyhedra $\left\{D_{\text {om }}, i \subseteq Y_{a} \times U, i \in Q\right\}$ is given, which covers the whole set $Y_{a} \times U$, i.e., $\cup_{i \in Q} D_{\text {or }}, i=Y_{a} \times U$. $D_{\text {om }}, i$ is defined through $r_{i}$ linear inequalities, i.e., $\operatorname{Dom}_{a, i}=\left\{\left(y_{a}, u\right) \in Y_{a} \times U: G_{i}^{y_{a}} y_{a}+G_{i}^{u} u \leq G_{i}\right\}$, with $G_{i}^{y_{a}} \in \mathbb{R}^{r_{i} \times p}, G_{i}^{u} \in \mathbb{R}^{r_{i} \times m}$ and $G_{i} \in \mathbb{R}^{r_{i}}$.

Mode $i \in Q$ is active as long as $\left(y_{a}, u\right)$ keeps evolving within $\operatorname{Dom}_{a, i}$ and a transition to mode $j \neq i \in Q$ occurs as soon as $\left(y_{a}, u\right)$ exits $D_{o m}, i$ and enters into $D_{o m}, j$ (endogenous switching).

Assumption 2 The switched affine system (1) admits a unique solution from any initial state.

Note that the considered switched system can be rephrased in the hybrid automata framework described in [30], where a precise notion of execution is given and conditions for well-posedness (existence and uniqueness) are mentioned. Moreover, if the collection $\left\{D_{\text {om }}, i, i \in Q\right\}$ is a polyhedral subdivision of $Y_{a} \times U^{1}$, then the SA system reduces to a standard piecewise affine system.

Remark 1 If the transition condition depends on the state $\xi_{a}$, then one can include $\xi_{a}$ as output variable to get back to the considered modeling framework where domains are defined as a function of the output (and input).

\section{System reduction: an approach based on BT}

The proposed procedure unfolds into the following steps: 1) the SA system is rewritten as a SL system with state reset (Section 3.1); 2) a reduced order model of the SL system is introduced by first applying BT to the continuous dynamics in each mode (Section 3.2), and then introducing appropriate maps for the reset of the reduced continuous state component when a mode transition occurs (Section 4); 3) the output of the SA system is reconstructed based on the reduced SL system output (Section 3.3).

\footnotetext{
1 This requires that each polyhedron $D_{0} m_{a, i}$ is of dimension $p+m$, and the intersection $\operatorname{Dom}_{a, i} \cap D_{o} m_{a, j}, i \neq j$, is either empty or a common proper face of both polyhedra.
} 


\subsection{Reformulation as a SL system with state reset}

We next build a SL system with state reset that is equivalent to the original SA system, in that $\left(\xi_{a}, q_{a}\right)$ and $y_{a}$ can be recovered exactly from the state and output variables of the SL system.

Let $y \in Y=Y_{a}$, and $\xi \in \Xi=\Xi_{a}$ evolve according to linear dynamics that depend on the operating mode $q \in Q$ as follows:

$$
\mathcal{S}:\left\{\begin{array}{l}
\dot{\xi}(t)=\mathcal{A}_{q} \xi(t)+\mathcal{B}_{q} u(t) \\
y(t)=\mathcal{C}_{q} \xi(t)
\end{array}\right.
$$

Set $\bar{y}_{a, q}=\mathcal{C}_{q} \bar{\xi}_{a, q}+g_{q}$, where $\bar{\xi}_{a, q}=-\mathcal{A}_{q}^{-1} f_{q}$, with $\mathcal{A}_{q}$ invertible by Assumption 1. A transition from mode $i \in$ $Q$ to mode $j \in Q$ occurs as soon as $\left(y+\bar{y}_{a, i}, u\right)$ exits $D_{\text {om }}$ and enters Dom , where Dom $_{q}=\operatorname{Dom}_{a, q}, q \in Q$. When a discrete transition from mode $i \in Q$ to mode $j \in Q$ occurs at time $t^{-}$, then, $\xi$ is reset as follows

$$
\xi(t)=\xi\left(t^{-}\right)+\Delta_{j i}^{\xi}, \quad \text { with } \Delta_{j i}^{\xi}=\bar{\xi}_{a, i}-\bar{\xi}_{a, j} .
$$

Proposition 1 Suppose that the $S A$ and $S L$ systems are initialized with initial conditions $\xi_{a}(0), q_{a}(0)$, and $\xi(0)=\xi_{a}(0)-\bar{\xi}_{a, q_{a}(0)}, q(0)=q_{a}(0)$, respectively, and are both fed by the same input $u(t), t \in[0, \mathcal{T}]$. Then, the executions of $\xi_{a}, q_{a}$ and $y_{a}$ over $[0, \mathcal{T}]$ can be recovered from those of $\xi, q$ and $y$ as follows:

$$
\begin{aligned}
& q_{a}(t)=q(t), \\
& \xi_{a}(t)=\xi(t)+\bar{\xi}_{a, q(t)}, \\
& y_{a}(t)=y(t)+\bar{y}_{a, q(t)} .
\end{aligned}
$$

Remark 2 The reset condition (3) is such that $\xi_{a}$ reconstructed from $\xi$ according to (4) is continuous. Continuity of $\xi_{a}$ is generally not guaranteed if $\xi$ is approximated through a reduced order model of the SL system.

\subsection{Reduction of the SL system}

A reduced order model of the SL system with state reset defined in Section 3.1 can be obtained by applying BT with the state residualization approach [4], to each single linear dynamics in (2). If the mode of the system were fixed, then, BT would be effective in reproducing the response $y$, at least in the long run, when the ZIR has vanished.

We associate with each mode $q \in Q$ a reduced model of order $n_{r, q}<n$ :

$$
\mathcal{S}_{r}:\left\{\begin{array}{l}
\dot{x}_{r, q}(t)=A_{r, q} x_{r, q}(t)+B_{r, q} u(t) \\
\hat{y}(t)=C_{r, q} x_{r, q}(t)+D_{r, q} u(t)
\end{array}\right.
$$

and define transitions between modes, say from mode $i$ to mode $j$, by evaluating when $\left(\hat{y}+\bar{y}_{a, i}, u\right)$ exits from domain $D_{o m}$ and enters into $D_{o m}$. Indeed, $\hat{y}+\bar{y}_{a, i}$ represent the output $y_{a}$ reconstructed using (4). As for the state reset map (3) associated with a mode transition from $i \in Q$ to $j \in Q$, we shall reformulate it as

$$
x_{r, j}(t)=L_{j i} x_{r, i}\left(t^{-}\right)+M_{j i} u\left(t^{-}\right)+N_{j i} \Delta_{j i}^{\xi},
$$

where $x_{r, i}\left(t^{-}\right) \in \mathbb{R}^{n_{r, i}}$ is the value of the reduced state in mode $i$, prior to the transition to mode $j, x_{r, j}(t) \in \mathbb{R}^{n_{r, j}}$ is the updated reduced state value, and $L_{j i}, M_{j i}, N_{j i}$ are matrices of appropriate dimensions. In Section 4, we present different methods to define them.

\subsection{Reconstruction of the SA system output}

The output of the SA system is reconstructed based on (4) using the output $\hat{y}$ of the SL reduced system as an estimate of the output $y$ of the SL system. This leads to

$$
\hat{y}_{a}(t)=\hat{y}(t)+\bar{y}_{a, q(t)} \text {. }
$$

\section{State reset maps: alternative choices}

In this section we introduce different reset maps that can be used for the approximate model. The choice of the reset map is of utter importance, since it strongly affects the quality of the approximated solution.

\subsection{Preliminary definitions}

Consider a transition from mode $i \in Q$ to mode $j \in Q$. One can determine an expression for $\hat{\xi}_{j}$, representing the SL system state associated with mode $j$ as reconstructed from the reduced state $x_{r, i}$.

Recall first that $\hat{\xi}_{i}$ can be obtained by applying the balanced transformation matrix $T_{i}$ to the reconstructed continuous state $\hat{x}_{i}$ of the SL system, i.e., $\hat{\xi}_{i}=T_{i}^{-1} \hat{x}_{i}$. In turn, $\hat{x}_{i}$ can be reconstructed as $\hat{x}_{i}=\left[x_{r, i}^{\prime} x_{n r, i}^{\prime}\right]^{\prime}$, where $x_{n r, i}$ is the part of the state that is neglected in the reduced model (5), and that can be recovered as a function of $x_{r, i}$ and $u$ by assuming an equilibrium condition in the original not-reduced linear dynamics (BT with state residualization) [4]. This leads to:

$$
\hat{x}_{i}=H_{i} x_{r, i}+K_{i} u,
$$

where $H_{i}$ and $K_{i}$ are suitable defined matrices [24]. Plugging the expressions of $\hat{\xi}_{i}$ and $\hat{x}_{i}$ into (3), yields

$$
\begin{aligned}
\hat{\xi}_{j}(t) & =\hat{\xi}_{i}\left(t^{-}\right)+\Delta_{j i}^{\xi} \\
& =T_{i}^{-1} H_{i} x_{r, i}\left(t^{-}\right)+T_{i}^{-1} K_{i} u\left(t^{-}\right)+\Delta_{j i}^{\xi} .
\end{aligned}
$$

We next shall define the reset maps for the state of the reduced SL system when a mode transition occurs from $i \in Q$ at time $t^{-}$to $j \in Q$ at time $t$. 


\subsection{SR map: a reset map based on state reconstruction}

The State Reconstruction-based reset map (SR map for brevity) was proposed in [23] and relies the following idea: reconstruct the whole state $\hat{x}_{j}(t)$ in balanced form and then extract its first $n_{r, j}$ components corresponding to the reduced order state in mode $j$. In formulas, $x_{r, j}(t)=E_{n_{r, j}} \hat{x}_{j}(t)$, where $E_{n_{r, j}}$ is a matrix that extracts the first $n_{r, j}$ rows from $\hat{x}_{j}(t), n_{r, j}$ being the dimension of $x_{r, j}$ in mode $j$. Now, $\hat{x}_{j}(t)$ can be obtained as $\hat{x}_{j}(t)=T_{j} \hat{\xi}_{j}(t)$.Plugging the expression of $x_{r, j}(t)$ into the expression of $\hat{x}_{j}(t)$, and using (8), we finally obtain

$x_{r, j}(t)=E_{n_{r, j}} T_{j}\left(T_{i}^{-1} H_{i} x_{r, i}\left(t^{-}\right)+T_{i}^{-1} K_{i} u\left(t^{-}\right)+\Delta_{j i}^{\xi}\right)$.

Matrices $L_{j i}, M_{j i}$, and $N_{j i}$ can be obtained by direct comparison with (6). According to a similar reasoning, the system is initialized as follows

$q_{r}(0)=q_{a}(0)=q_{0}, x_{r, q_{0}}(0)=E_{n_{r, q_{0}}} T_{q_{0}}\left(\xi_{a}(0)-\bar{\xi}_{a, q_{0}}\right)$,

with the understanding that $\left(y_{a}(0), u(0)\right)$ is an interior point of $D_{o m} q_{0}$, for any admissible $u(0)$.

\subsection{OG map: a reset map to reproduce the output ZIR}

Model reduction techniques for asymptotically stable linear systems aim at finding a model that best reproduce the forced response of the system, while neglecting the ZIR. However, in SA systems, the system response depends on the mode transitions, which, in turn, depends on the continuous output behavior (forced plus ZIR). We here introduce a reset map that minimizes the $L^{2}$-norm of the error when reproducing the ZIR of the output $y$. As we shall see next, its expression depends on the Observability Gramians (OG) of the linear systems associated with the different modes.

In formulas, we set $x_{r, j}=\Psi_{j} \hat{\xi}_{j}$ and choose $\Psi_{j}$ so as to minimize

$$
J\left(\Psi_{j}\right)=\int_{0}^{+\infty}\left\|y_{z i r, j}(t)-\hat{y}_{z i r, j}(t)\right\|^{2} \mathrm{~d} t,
$$

where $y_{z i r, j}$ and $\hat{y}_{z i r, j}$ respectively denote the ZIR of the original linear dynamics (2) initialized with $\hat{\xi}_{j}$ and that of the reduced order dynamics (5) initialized with $x_{r, j}=\Psi_{j} \hat{\xi}_{j}$. The solution to this optimization problem can be found analytically as shown in Proposition 2, which proof can be found in [24].

Proposition 2 Suppose that the reduced order model (5) with $q=j$ is observable. Then, matrix $\Psi_{j}^{\star}$ minimizing (10) for any $\hat{\xi}_{j}$ is given by $\Psi_{j}^{\star}=\mathcal{W}_{r, o, j}^{-1} \mathcal{W}_{\times, j}$, where

$$
\begin{aligned}
& \mathcal{W}_{r, o, j}=\int_{0}^{+\infty}\left(e^{A_{r, j} t}\right)^{\prime} C_{r, j}^{\prime} C_{r, j} e^{A_{r, j} t} \mathrm{~d} t \\
& \mathcal{W}_{\times, j}=\int_{0}^{+\infty}\left(e^{\mathcal{A}_{j} t}\right)^{\prime} \mathcal{C}_{j}^{\prime} C_{r, j} e^{A_{r, j} t} \mathrm{~d} t .
\end{aligned}
$$

Remark 3 Note that the observability assumption in Proposition 2 is satisfied under mild conditions as detailed in [4].

Matrix $\mathcal{W}_{r, o, j}$ can be obtained by solving the Lyapunov equation

$$
A_{r, j} \mathcal{W}_{r, o, j}+\mathcal{W}_{r, o, j} A_{r, j}^{\prime}+C_{r, j}^{\prime} C_{r, j}=0
$$

while matrix $\mathcal{W}_{\times, j}$ is the solution to the Sylvester equation

$$
A_{r, j}^{\prime} \mathcal{W}_{\times, j}+\mathcal{W}_{\times, j} \mathcal{A}_{j}+C_{r, j}^{\prime} \mathcal{C}_{j}=0
$$

Now, plugging the expression $(8)$ for $\hat{\xi}_{j}(t)$ into $x_{r, j}=$ $\Psi_{j} \hat{\xi}_{j}$ and setting $\Psi_{j}=\Psi_{j}^{\star}$, we get

$$
x_{r, j}(t)=\Psi_{j}^{\star}\left(T_{i}^{-1} H_{i} x_{r, i}\left(t^{-}\right)+T_{i}^{-1} K_{i} u\left(t^{-}\right)+\Delta_{j i}^{\xi}\right)
$$

Matrices $L_{j i}, M_{j i}$, and $N_{j i}$ can be obtained by direct comparison of with (6). As for the system initialization, we set

$$
q_{r}(0)=q_{a}(0)=q_{0}, x_{r, q_{0}}(0)=\Psi_{q_{0}}^{\star}\left(\xi_{a}(0)-\bar{\xi}_{a, q_{0}}\right) .
$$

Instead of considering an infinite horizon when evaluating the ZIR output error, one can take into account the switching nature of the system and consider the error only during the finite horizon $[0, \tau]$. Correspondingly, the error function to be minimized becomes

$$
J_{\tau}\left(\Psi_{j}^{\tau}\right)=\int_{0}^{\tau}\left\|y_{z i r, j}(t)-\hat{y}_{z i r, j}(t)\right\|^{2} \mathrm{~d} t .
$$

The resulting optimal $\Psi_{j}^{\tau \star}$ matrix is given by $\Psi_{j}^{\tau \star}=$ $\mathcal{W}_{r, o, j}^{-1}(\tau) \mathcal{W}_{\times, j}(\tau)$, where

$$
\begin{aligned}
& \mathcal{W}_{r, o, j}(\tau)=\int_{0}^{\tau}\left(e^{A_{r, j} t}\right)^{\prime} C_{r, j}^{\prime} C_{r, j} e^{A_{r, j} t} \mathrm{~d} t \\
& \mathcal{W}_{\times, j}(\tau)=\int_{0}^{\tau}\left(e^{\mathcal{A}_{j} t}\right)^{\prime} \mathcal{C}_{j}^{\prime} C_{r, j} e^{A_{r, j} t} \mathrm{~d} t .
\end{aligned}
$$

The proof of this result is analogous to that in the infinite horizon case. Still, observability of the reduced order model (5) with $q=j$ is required for $\mathcal{W}_{r, o, j}$ to be invertible and Remark 3 applies.

The finite horizon quantities involved in the expression of $\Psi_{j}^{\tau \star}$ can be computed as

$$
\begin{aligned}
\mathcal{W}_{r, o, j}(\tau) & =\mathcal{W}_{r, o, j}-\int_{\tau}^{+\infty}\left(e^{A_{r, j} t}\right)^{\prime} C_{r, j}^{\prime} C_{r, j} e^{A_{r, j} t} \mathrm{~d} t \\
& =\mathcal{W}_{r, o, j}-\mathcal{W}_{r, o, j}^{(\tau, \infty)}, \\
\mathcal{W}_{\times, j}(\tau) & =\mathcal{W}_{\times, j}-\int_{\tau}^{\infty}\left(e^{\mathcal{A}_{j} t}\right)^{\prime} \mathcal{C}_{j}^{\prime} C_{r, j} e^{A_{r, j} t} \mathrm{~d} t \\
& =\mathcal{W}_{\times, j}-W_{\times, j}^{(\tau, \infty)},
\end{aligned}
$$

where $\mathcal{W}_{r, o, j}^{(\tau, \infty)}$ and $\mathcal{W}_{\times, j}^{(\tau, \infty)}$ can be obtained as the solution of the Lyapunov and Sylvester equations

$$
\begin{aligned}
& A_{r, j} \mathcal{W}_{r, o, j}^{(\tau, \infty)}+\mathcal{W}_{r, o, j}^{(\tau, \infty)} A_{r, j}^{\prime}+\left(e^{A_{r, j} \tau}\right)^{\prime} C_{r, j}^{\prime} C_{r, j} e^{A_{r, j} \tau}=0, \\
& A_{r, j}^{\prime} \mathcal{W}_{\times, j}^{(\tau, \infty)}+\mathcal{W}_{\times, j}^{(\tau, \infty)} \mathcal{A}_{j}+\left(e^{A_{r, j} \tau}\right)^{\prime} C_{r, j}^{\prime} \mathcal{C}_{j} e^{\mathcal{A}_{j} \tau}=0 .
\end{aligned}
$$


Note that well-posedness of the above equations is guaranteed by the fact that $A_{j}$ and $A_{r, j}$ are Hurwitz.

The matrices in the reset map (6) and the system initialization are analogous to the case of infinite horizon, but with $\Psi_{j}^{\tau \star}$ in place of $\Psi_{j}^{\star}$.

The choice for $\tau$ depends on the settling times of the different mode dynamics. A sensible choice is to set $\tau$ equal to the settling time of the neglected dynamics.

To distinguish between the two OG reset maps, we shall refer to the one with the infinite horizon as $\mathrm{OG}_{\infty}$ and the one with finite horizon $[0, \tau]$ as $\mathrm{OG}_{\tau}$.

\subsection{Variants that preserve the output continuity}

In certain application contexts, it may be desirable to preserve the continuity of the output of the original system. This is not guaranteed when adopting the reset maps defined above and motivates the derivations hereafter.

To get continuity, the value of the output $\hat{y}_{a}(t)$ reconstructed based on (7) before and after the reset should be identical. This leads to the following equation

$C_{r, j} x_{r, j}(t)+D_{r, j} u(t)+\bar{y}_{a, j}=C_{r, i} x_{r, i}\left(t^{-}\right)+D_{r, i} u\left(t^{-}\right)+\bar{y}_{a, i}$.

Under the assumption that the input $u$ is a continuous signal, and letting $\Delta_{j i}^{y}=\bar{y}_{a, i}-\bar{y}_{a, j}$, this simplifies to

$$
C_{r, j} x_{r, j}(t)=C_{r, i} x_{r, i}\left(t^{-}\right)+\left(D_{r, i}-D_{r, j}\right) u\left(t^{-}\right)+\Delta_{j i}^{y} .
$$

The values of $x_{r, j}(t)$ that satisfy the above condition can be expressed as $x_{r, j}(t)=\tilde{x}_{r, j}(t)+w_{j}$, with

$\tilde{x}_{r, j}(t)=C_{r, j}^{\dagger}\left(C_{r, i} x_{r, i}\left(t^{-}\right)+\left(D_{r, i}-D_{r, j}\right) u\left(t^{-}\right)+\Delta_{j i}^{y}\right)$

where $C_{r, j}^{\dagger}$ is the pseudo-inverse of $C_{r, j}$ and $w_{j} \in \mathbb{R}^{n_{r, j}}$ is in the null space of $C_{r, j}$, here denoted as $\operatorname{ker}\left(C_{r, j}\right)$. If $\operatorname{ker}\left(C_{r, j}\right) \neq\{0\}$, we have some degrees of freedom to spend and we can choose $w_{j}$ so that the resulting value for $x_{r, j}(t)$ best matches some given reference value $\bar{x}_{r, j}(t)$. If instead $\operatorname{ker}\left(C_{r, j}\right)=\{0\}$, then, $w_{j}=0$, and the reset matrices are derived by a direct comparison with (6).

Let us consider now the case when $\operatorname{ker}\left(C_{r, j}\right) \neq\{0\}$. If we let $\left\{v_{1}, v_{1}, \ldots, v_{n_{v, j}}\right\}$ be a basis of $\operatorname{ker}\left(C_{r, j}\right)$, and set $V_{j}=\left[\begin{array}{llll}v_{1} & v_{2} & \ldots & v_{n_{v, j}}\end{array}\right]$, then, $w_{j}=V_{j} \alpha$ with $\alpha \in \mathbb{R}^{n_{v, j}}$ and we can select $\alpha$ by solving the least squares problem

$$
\alpha^{\star}=\arg \min _{\alpha}\left\|\tilde{x}_{r, j}(t)+V_{j} \alpha-\bar{x}_{r, j}(t)\right\|
$$

which leads to $\alpha^{\star}=V_{j}^{\dagger} \bar{x}_{r, j}(t)$, since it holds that $V_{j}^{\dagger} C_{r, j}^{\dagger}=0$. We then finally have:

$$
x_{r, j}(t)=\tilde{x}_{r, j}(t)+V_{j} V_{j}^{\dagger} \bar{x}_{r, j}(t),
$$

which, depending on the chosen $\bar{x}_{r, j}(t)$ leads to different expressions for the matrices $L_{j i}, M_{j i}$, and $N_{j i}$ in the reset map (6).

If we adopt the expression in the SR map (9) for $\bar{x}_{r, j}(t)$, then we can define the Continuous State Reconstructionbased reset map (CSR map). If we instead set $\bar{x}_{r, j}(t)$ equal to the $\mathrm{OG}_{\infty}$ map expression (11), we obtain the Continuous Observability Gramian-based map with infinite horizon $\left(\mathrm{COG}_{\infty}\right.$ map). Analogously, we can define the Continuous Observability Gramian-based map with finite horizon $[0, \tau]\left(\mathrm{COG}_{\tau}\right)$.

As for the initialization, $q(0)=q_{a}(0)=q_{0}$, whereas the value for $x_{r, q_{0}}(0)$ is obtained by setting the value of the output $\hat{y}_{a}(0)$ reconstructed based on $(7)$ equal to that of $y_{a}(0)$ obtained based on the system initialization. This leads to the following equation

$$
C_{r, q_{0}} x_{r, q_{0}}(0)+D_{r, q_{0}} u(0)+\bar{y}_{a, q_{0}}=y_{a}(0),
$$

where $y_{a}(0)$ is given by the initial conditions of the system, i.e., $y_{a}(0)=\mathcal{C}_{q_{a}} \xi_{a}(0)+g_{q_{0}}$.

From this equation, by following similar steps than those used for deriving (13), we get that

$x_{r, q_{0}}(0)= \begin{cases}\tilde{x}_{r, q_{0}}(0), & \operatorname{ker}\left(C_{r, j}\right)=\{0\} \\ \tilde{x}_{r, q_{0}}(0)+V_{q_{0}} V_{q_{0}}^{\dagger} \bar{x}_{r, q_{0}}(0), & \operatorname{ker}\left(C_{r, j}\right) \neq\{0\},\end{cases}$

where we set $\tilde{x}_{r, q_{0}}(0)=C_{r, q_{0}}^{\dagger}\left(-D_{r, q_{0}} u(0)-\bar{y}_{a, q_{0}}+y_{a}(0)\right)$, and $\bar{x}_{r, q_{0}}(0)$ is the initialization of the $\mathrm{SR}, \mathrm{OG}_{\infty}$, or $\mathrm{OG}_{\tau}$ reset map.

\section{A randomized method for order selection}

In [23], following an approach that is quite standard for linear systems [4], a threshold value $\gamma$ is chosen, and the order of the reduced SL system (5) in mode $q \in Q$ is set equal to

$$
n_{r, q}=\min \left\{i \in\{1,2, \ldots, n\}: \psi_{q}(i)<\gamma\right\},
$$

where $\psi_{q}:\{1,2, \ldots n\} \rightarrow[0,1)$ is given by $\psi_{q}(i)=$ $1-\sum_{j=1}^{i} \sigma_{j, q} / \sum_{j=1}^{n} \sigma_{j, q}, \sigma_{1, q} \geq \sigma_{2, q} \geq \cdots \geq \sigma_{n, q}$ being the HSVs of the SL system dynamics (2) in mode $q$. Our goal here is to introduce a sound method for making an appropriate selection of the threshold value $\gamma$, when the input $u$ is stochastic and one has to verify a property that depends on the behavior of the SA system output $y_{a}$ along a finite time horizon $\mathcal{T}$. For the resulting stochastic hybrid system and its executions to be well-defined according to the notion in [16], we shall assume in the following that input $u$ is a white noise with a given power spectral density.

A randomized method for order selection is proposed, which involves feeding the candidate reduced order models and the system with the same realizations of the stochastic input and comparing their outputs over $\mathcal{T}$. If 
the number of realizations is appropriately chosen, then the quality of the model assess over them generalizes to the unseen instances, except for a set of a-priori defined probability $\epsilon$. Notably, this can be reinterpreted as an $\epsilon$-robust assessment result.

Let us denote by $\Gamma$ the (finite) set of possible threshold values $\gamma$, those that result in a different choice for $\left\{n_{r, q}, q \in Q\right\}$, and by $\hat{y}_{a}^{\gamma}$ the estimate of $y_{a}$ obtained through the reduced SL system when the threshold value is set equal to $\gamma$.

The approximation error can be quantified through a function $d_{\mathcal{T}}(\cdot, \cdot)$ that maps each pair of trajectories $y_{a}(t), t \in \mathcal{T}$, and $\hat{y}_{a}^{\gamma}(t), t \in \mathcal{T}$, into a positive real number $d_{\mathcal{T}}\left(y_{a}, \hat{y}_{a}^{\gamma}\right)$ that represents the extent to which the output $y_{a}$ of the SA system differs from its estimate $\hat{y}_{a}^{\gamma}$ along the time horizon $\mathcal{T}$. Function $d_{\mathcal{T}}(\cdot, \cdot)$ satisfies $d_{\mathcal{T}}\left(y_{a}, \hat{y}_{a}^{\gamma}\right)=0$ if $\gamma=0$, since in that case no reduction is performed and, hence, $\hat{y}_{a}^{\gamma}(t)=y_{a}(t), t \in \mathcal{T}$.

In order to make an appropriate selection of $\gamma$, we adopt the notion of approximate simulation in $[3,9,17,28]$ to assess the quality of the reduced order model with threshold value $\gamma$. This involves computing the maximal value $\rho_{\gamma}^{\star}$ taken by $d_{\mathcal{T}}\left(y_{a}, \hat{y}_{a}^{\gamma}\right)$ over all realizations of the stochastic input $u(t)$ and the (possibly) stochastic initialization $\xi_{a}(0)$ of the SA system, except for a set of probability at most $\epsilon \in(0,1)$. An 'optimal' value for $\gamma$ can then be chosen by inspecting the values of $\rho_{\gamma}^{\star}$ as a function of $\gamma \in \Gamma$ and selecting the appropriate compromise between quality of the approximation and tractability of the resulting reduced order model.

More precisely, we introduce the following family of chance-constrained optimization problems (CCPs) parametrized by $\gamma \in \Gamma$ :

$$
\begin{aligned}
& C C P_{\gamma}: \min _{\rho} \rho \\
& \quad \text { subject to: } \mathbb{P}\left\{d_{\mathcal{T}}\left(y_{a}, \hat{y}_{a}^{\gamma}\right) \leq \rho\right\} \geq 1-\epsilon .
\end{aligned}
$$

By directly inspecting the solution of (15) as a function of $\gamma$, one can then select the appropriate compromise between accuracy and simplicity of the model, respectively expressed through $\rho_{\gamma}^{\star}$, and $n_{r, q}, q \in Q$, in (14).

Remark 4 As argued in [3], the directional Hausdorff distance $d_{\mathcal{T}}\left(y_{a}, \hat{y}_{a}^{\gamma}\right)=\sup _{t \in \mathcal{T}} \inf _{\tau \in \mathcal{T}}\left\|y_{a}(t)-\hat{y}_{a}^{\gamma}(\tau)\right\|$ is a sensible choice for $d_{\mathcal{T}}\left(y_{a}, \hat{y}_{a}^{\gamma}\right)$ when performing probabilistic verification, e.g., when estimating of the probability that $y_{a}$ will enter some set within $\mathcal{T}$.

Solving CCPs like (15) is known to be difficult, and even NP-hard in some cases, [5].We then head for an approximate solution where instead of considering all the possible realizations for the stochastic uncertainty, we consider only a finite number $N$ of them called "scenarios", extracted at random, and treat them as if they were the only admissible uncertainty instances. This leads to the formulation of Algorithm 1, where the chance-constrained solution is determined based on the extracted scenarios and a empirical violation parameter $\eta \in(0, \epsilon)$. Notably, in Proposition 3 it is proven that,

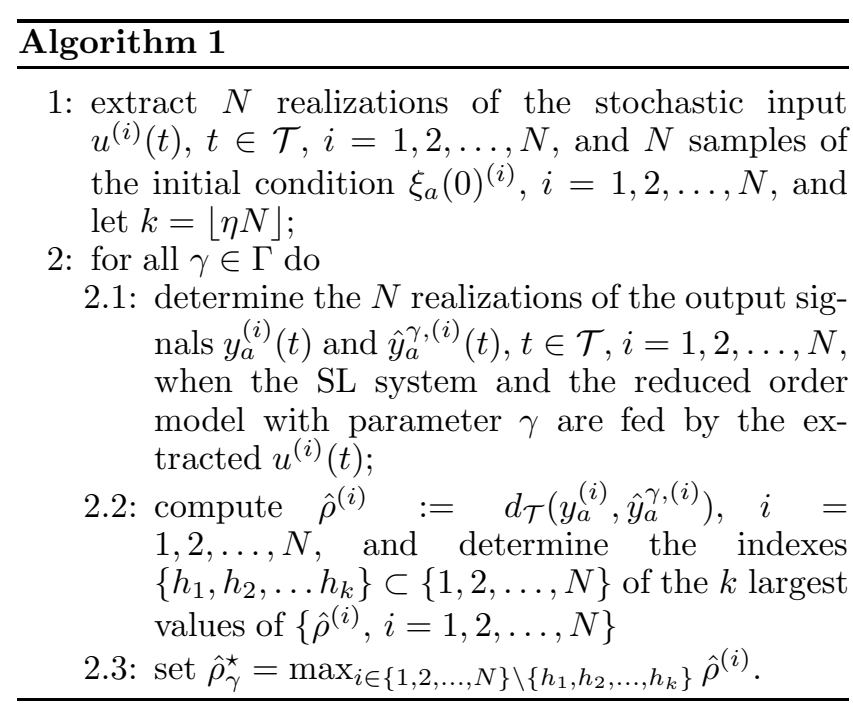

if the number $N$ of extractions is appropriately chosen, the obtained estimate of $\rho_{\gamma}^{\star}$ is chance-constrained feasible, uniformly with respect to $\gamma \in \Gamma$, with a-priori specified (high) probability. The proof of Proposition 3 can be found in [24], and rests on results from the scenario approach $[5,6]$.

Proposition 3 Select a confidence parameter $\beta \in$ $(0,1)$, and an empirical violation parameter $\eta \in(0, \epsilon)$. If $N$ satisfies

$$
\sum_{i=0}^{\lfloor\eta N\rfloor}\left(\begin{array}{c}
N \\
i
\end{array}\right) \epsilon^{i}(1-\epsilon)^{N-i} \leq \frac{\beta}{|\Gamma|},
$$

where $|\Gamma|$ denotes the cardinality of $\Gamma$, then, the solution $\hat{\rho}_{\gamma}^{\star}, \gamma \in \Gamma$, to Algorithm 1 satisfies $\mathbb{P}\left\{d_{\mathcal{T}}\left(y_{a}, \hat{y}_{a}^{\gamma}\right) \leq \hat{\rho}_{\gamma}^{\star}\right\} \geq$ $1-\epsilon, \forall \gamma \in \Gamma$, with probability at least $1-\beta$.

If we discard the confidence parameter $\beta$ for a moment, this proposition states that for any $\gamma \in \Gamma$, the randomized solution $\hat{\rho}_{\gamma}^{\star}$ obtained through Algorithm 1 is feasible for the chance-constrained problem (15). As $\eta$ tends to $\epsilon, \hat{\rho}_{\gamma}^{\star}$ approaches the desired optimal chance constrained solution $\rho_{\gamma}^{\star}$. In turn, the computational effort grows unbounded since $N$ scales as $\frac{1}{\epsilon-\eta},[5]$, therefore, the value for $\eta$ depends in practice from the available computational resources. As for $\beta$, one should note that $\hat{\rho}_{\gamma}^{\star}$ is a random quantity that depends on the randomly extracted input realizations and initial conditions. It may happen that the extracted samples are not representative enough, in which case the size of the violation set will be larger than $\epsilon$. Parameter $\beta$ controls the probability that this happens and the final result holds with probability $1-\beta . N$ satisfying (16) depends logarithmically on $|\Gamma| / \beta,[5]$, so that $\beta$ can be chosen as small as $10^{-10}$ (and, hence, $1-\beta \simeq 1$ ) without growing significantly $N$.

Interestingly, the guarantees provided by Proposition 3 are valid irrespectively of the underlying probability distribution of the input, which may even not be known ex- 
plicitly, e.g., when running Algorithm 1 with historical time series as realizations of the stochastic input $u$.

Remark 5 Note that even in the case of stable continuous dynamics, switching can produce unstable behaviors. However, if some reduced order model presents an unstable behavior, which makes the distance between $y_{a}$ and $\hat{y}_{a}^{\gamma}$ large, that model is not selected.

\section{Numerical example}

In this section, a multi-room heating system with a switching control policy is presented. The example is inspired to a benchmark for hybrid system verification presented in [7].

Consider the problem of controlling the temperature in a number of rooms of a building. Each room has one heater, but there is a constraint on the number of heaters in the building that can be "active" (i.e., that can be used and turned on if needed) at the same time. Differently from the original benchmark in [7], we model also the dynamics of the heaters.

The temperature $T_{i}$ in a room $i \in\left\{1, \ldots, N_{r}\right\}$ depends on $T_{i}$ itself, on the temperature of the adjacent rooms $T_{j}$ with $j \neq i$, on the outside temperature $T_{\text {ext }}$, and on $h_{i}$, a boolean variable that is 1 when the heater is on in room $i$, and 0 otherwise. The heat transfer coefficient between room $i$ and room $j$ is $k_{i j}$, and the one between room $i$ and the external environment is $k_{e, i}$. We assume that the heat exchange is symmetric, i.e., $k_{i j}=k_{j i}$. Rooms $i$ and $j$ are adjacent when $k_{i j}>0$, otherwise $k_{i j}=0$.

The volume of the room is $V_{i}$, and the wall surface between room $i$ and room $j$ is $S_{r, i j}$, while that between room $i$ and the environment is $S_{e, i}$. Air density and heat capacity are $\rho_{a}=1.225 \mathrm{~kg} / \mathrm{m}^{3}$ and $c=1005 \mathrm{~J} /(\mathrm{kg} \mathrm{K})$, respectively. Letting $\phi_{i}=\rho_{a} c V_{i}$, we can formulate the following dynamic model for room $i$ and its heater:

$$
\begin{aligned}
\phi_{i} \dot{T}_{i} & =\sum_{j \neq i} S_{r, i j} k_{i j}\left(T_{j}-T_{i}\right)+S_{e_{i}} k_{e, i}\left(T_{\text {ext }}-T_{i}\right)+\kappa_{i} \theta_{i} \\
\tau_{h, i} \dot{\theta}_{i} & =-\theta_{i}+h_{i} \cdot p_{i}-\chi_{i} T_{\text {ext }}
\end{aligned}
$$

which is an affine system, with $\kappa_{i}$ representing the maximum heat flow rate that the heater can provide, while $p_{i} \in\{0,1\}$ is a binary variable indicating if the heater is active in room $i\left(p_{i}=1\right)$ or not $\left(p_{i}=0\right)$. The heater dynamics is represented by a first-order system with a time constant $\tau_{h, i}$. If we neglect the term $-\chi_{i} T_{\text {ext }}$ in the heater dynamics and set $h_{i}=p_{i}=1$, the heater state variable $\theta_{i}$ will tend to 1 so that the heater will provide its maximum heat flow rate $\kappa_{i}$ to the room when it is active and on. The term $-\chi_{i} T_{\text {ext }}$ is introduced to account for the influence of the external temperature on the heating system. Notice that $p_{i}=1$ just indicates that the heater is active in room $i$, while $h_{i}$ is the variable that indicates whether it is actually turned on $\left(h_{i}=1\right)$. The physical nature of the considered system is not switching. However, the switching control policy presented in [7] is used to control the temperature in the rooms.

A room policy decides whether to switch the heater on in the room: each room has a thermostat that switches the heater on if $T_{i} \leq$ on $_{i}$, and off when $T_{i} \geq$ off $_{i}$.

A building policy decides and limits the number of heaters that are jointly active, by setting the constraint $\sum_{i=1}^{N_{r}} p_{i}=\bar{P}$, with $\bar{P} \leq N_{r}$. The heater of room $i$ is turned active, and the heater of room $j$ becomes not active when: 1) the heater of some room, say room $i$, is not active, i.e., $\left.p_{i}=0,2\right)$ room $j$ is adjacent to room $i$ and has an active heater, i.e., $\left.p_{j}=1,3\right)$ temperature $T_{i} \leq$ get $_{i}$, and 4) the difference $T_{j}-T_{i} \geq \operatorname{dif}_{i}$.

Each room is identified by an integer index, and whenever a room has more than one adjacent room fulfilling the above condition, the heater is always set active in the room with higher index.

In the following we consider $N_{r}=4$ adjacent rooms, with the constraint that only $\bar{P}=3$ heaters can be active at the same time. The values of the physical system parameters for the considered instance of the problem are reported in Table 1 . The external temperature $T_{\text {ext }}$ is modeled as a sinusoidal source of period 24 hours with an offset of $4^{\circ} \mathrm{C}$, affected by an additive white noise. Note that the resulting stochastic hybrid system and its execution are still well-defined (see [18]).

We assume deterministic initial conditions, i.e., $T_{i}(0)=$ $20, \theta_{i}(0)=0, i=1, \ldots, N_{r}, h(0)=p(0)=\left[\begin{array}{llll}0 & 1 & 1 & 1\end{array}\right]^{\prime}$. The condition that only 3 out of 4 heaters are active at the same time is satisfied by $p(0)$. As for the control policy parameters, we set off $i=21$, on $_{i}=20$, get $_{i}=18$, $\operatorname{dif}_{i}=1$, with $i=1, \ldots, N_{r}$. Due to the switching policy, the control system can be described as a SA system with continuous state $\xi_{a}=\left[T^{\prime} \theta^{\prime}\right]^{\prime}$, input $u=T_{\text {ext }}$, and output $y_{a}=T$ :

$$
\left\{\begin{array}{l}
\dot{\xi}_{a}=\mathcal{A} \xi_{a}+\mathcal{B} u+f_{q_{a}} \\
y_{a}=\mathcal{C} \xi_{a} .
\end{array}\right.
$$

As for the mode $q_{a}$, it is identified by the values of the binary variables $h_{i}$ and $p_{i}$, which determine the affine term $f_{q_{a}}$ entering the dynamics of $\xi_{a}$. The polyhedral sets $\operatorname{Dom}_{a, q_{a}}$ are determined by the building and room control policies through the chosen thresholds. Note that only the affine term $f_{q_{a}}$ in (17) depends on the discrete mode $q_{a} \in Q$, while the state-space matrices $(\mathcal{A}, \mathcal{B}, \mathcal{C})$ are constant. Therefore, the BT can be computed only once, and applied identically for each discrete mode. Still, when selecting the order of the reduced model

Table 1

The multi-room physical system parameters.

\begin{tabular}{cl|cl|cl}
$S_{r, i j}$ & $12 \mathrm{~m}^{2}$ & $k_{e, i}$ & $1 \mathrm{~W} /\left(\mathrm{m}^{2} \mathrm{~K}\right)$ & $\tau_{h, 3}$ & $45.00 \mathrm{~s}$ \\
$S_{e, i}$ & $24 \mathrm{~m}^{2}$ & $\kappa_{1}$ & 0.373 & $\tau_{h, 4}$ & $47.25 \mathrm{~s}$ \\
$V_{i}$ & $48 \mathrm{~m}^{3}$ & $\kappa_{2}$ & 0.395 & $\chi_{1}$ & $1.0 \times 10^{-4}$ \\
$\phi_{i}$ & $59094 \mathrm{~J} / \mathrm{K}$ & $\kappa_{3}$ & 0.417 & $\chi_{2}$ & $2.0 \times 10^{-4}$ \\
$k_{12}$ & $2 \mathrm{~W} /\left(\mathrm{m}^{2} \mathrm{~K}\right)$ & $\kappa_{4}$ & 0.439 & $\chi_{3}$ & $3.0 \times 10^{-4}$ \\
$k_{23}$ & $5 \mathrm{~W} /\left(\mathrm{m}^{2} \mathrm{~K}\right)$ & $\tau_{h, 1}$ & $40.50 \mathrm{~s}$ & $\chi_{4}$ & $4.0 \times 10^{-4}$ \\
$k_{34}$ & $2 \mathrm{~W} /\left(\mathrm{m}^{2} \mathrm{~K}\right)$ & $\tau_{h, 2}$ & $42.75 \mathrm{~s}$ & & \\
\hline
\end{tabular}


one should consider the impact of the selected order on the switched system approximation, which involves also mode transitions. Using standard approaches for the order selection, as the one used in [23] relying on classical HSV analysis, can be misleading. Indeed, the obtained HSVs are $\sigma_{1}=0.993, \sigma_{2}=0.026, \sigma_{3}=0.001$, $\sigma_{4}=4.514 \times 10^{-5}, \sigma_{5}=1.897 \times 10^{-6}, \sigma_{6}=6.995 \times 10^{-7}$, $\sigma_{7}=1.805 \times 10^{-8}, \sigma_{8}=3.534 \times 10^{-10}$. The HSV analysis suggests that most of the dynamics can be caught by reducing the continuous dynamics of the SA system to a first-order one. Indeed, computing the distance $\psi\left(n_{r}\right)$ used in [23] results in $\psi(1) \cdot 100=2.64 \%$.

Care has to be taken when applying HSV analysis to the context of SA systems. In fact, classical BT techniques are typically based on the assumption that the ZIR of the system can be neglected since it vanishes in an asymptotically stable linear system, a fact that notoriously does not always hold when dealing with switching systems. Moreover, HSV analysis does not take into account the impact of the reset map.

The multi-room control system is next reduced by means of the constructive methodology proposed in this paper, and the randomized approach for order selection based on the directional Hausdorff distance evaluated over a finite horizon $\mathcal{T}=[0,200]$ min is applied. In particular, we set $\epsilon=0.1$ in the CCP (15) and solve it via Algorithm 1. The number of extractions in Algorithm 1 is $N=778$ and is obtained through the implicit formula (16) with $\eta=0.05, \beta=10^{-6}$ and $|\Gamma|=7$.

Since we adopt the same order for the reduced dynamics in each mode, 7 model order reductions are examined, and, according to Proposition 3, the results on the quality assessment of the reduced order models hold with probability $1-10^{-6}$.

The length $\tau$ of the finite horizon $[0, \tau]$ adopted in $\mathrm{OG}_{\tau}$ and $\mathrm{COG}_{\tau}$ is set to the settling time of the neglected dynamics. Equation (14) maps each threshold value $\gamma \in \Gamma$ into the order $n_{r, q}$ of the reduced dynamics within mode $q \in Q$ of the SL system with state reset. In this example, we adopt the same order for the reduced dynamics in each mode. Hence, we can simplify the notation to $n_{r}$, dropping the dependence from mode $q$. The values for $\hat{\rho}_{\gamma}^{\star}$ obtained with the different reset methods are presented in Figure 1 as a function of $n_{r}$. Some interesting

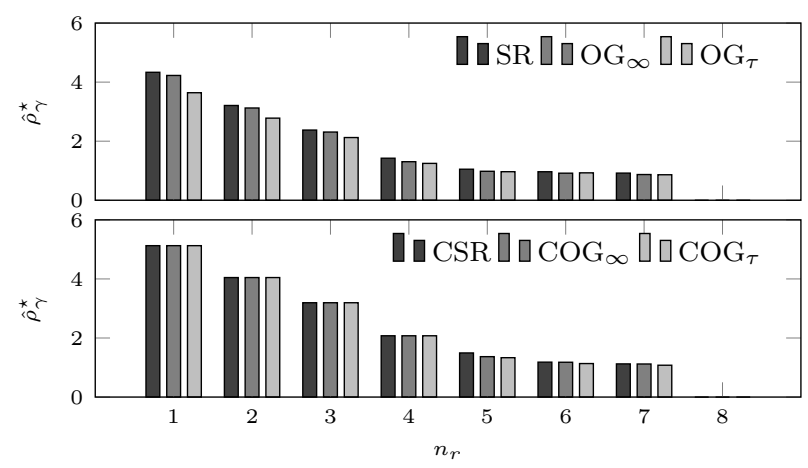

Figure 1. Performance of different reduced models as a function of the order $n_{r}$ and of the adopted reset maps. considerations can be made by analyzing the results presented in Figure 1. First of all, one can compare the reset maps that do preserve continuity with those that do not. The plots in Figure 1 show that preserving continuity leads to worse performance in terms of accuracy of the approximation. This holds despite of the fact that, for the maps that do not preserve output continuity, a drastic order reduction may yield discontinuities in the state reset that possibly produce chattering behaviors. Furthermore, Figure 1 shows that the OG reset maps exhibit better performance with respect to the SR maps. In particular, for the $\mathrm{OG}_{\tau}$ map $\hat{\rho}_{\gamma}^{\star}$ is reduced on average over $n_{r}$ by $10.03 \%$ in the discontinuous map case, and by $2.68 \%$ in the continuous map case.

Notice also that when a reduced order $n_{r} \leq p=4$ is used and the output continuity is enforced, then, the same results are obtained with the different reset maps. This is due to the fact that whenever $n_{r} \leq p$, there are no degrees of freedom left by the continuity constraint to match the originally introduced SR or OG reset maps (see the derivations in Section 4.4), so that all maps just enforce continuity and become identical.

From the randomized analysis in Figure 1, it appears that one can push the reduction up to a fifth order without significantly deteriorating the accuracy of the model when the goal of the approximation is the analysis of reachability properties for which the directional Hausdorff distance is a suitable accuracy measure. Reducing the system to a first-order approximation as suggested by the analysis based on the HSV only would instead result in a quite significant degradation of the reduced model performance.

\section{Extension to SA systems with DT}

The approach that we proposed in Section 6 for model order reduction can also be applied to the case when the mode transitions of the SA system are subject to a DT constraint, which means that a transition from mode $i \in Q$ to mode $j \neq i \in Q$ is enabled when $\left(y_{a}, u\right)$ exits

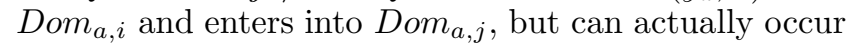
only if a certain minimum amount of time $\bar{\delta}_{i} \in \mathbb{R}^{+}$(the so-called dwell time) has elapsed. Note that DT can be present in a system for two different reasons: either is due to an intrinsic characteristic of the system that presents some delay/inertia when commuting, or it is introduced when designing a control strategy, as in DT switching control, see e.g. [15,20].

An extension of the SA modeling framework is needed if a DT constraint is present. If we start from a SA system of the form (1), we can introduce the DT constraint as described next. DT can be accounted for by adding to each mode a continuous state variable $\delta \in \mathbb{R}$ that represents a clock with the dynamics of an integrator. 
The dynamics (1) then is augmented as follows:

$$
\begin{aligned}
& {\left[\begin{array}{c}
\dot{\xi}_{a}(t) \\
\dot{\delta}(t)
\end{array}\right]=\left[\begin{array}{cc}
\mathcal{A}_{q_{a}} & 0 \\
0 & 0
\end{array}\right]\left[\begin{array}{c}
\xi_{a}(t) \\
\delta(t)
\end{array}\right]+\left[\begin{array}{cc}
\mathcal{B}_{q_{a}} & 0 \\
0 & 1
\end{array}\right]\left[\begin{array}{c}
u(t) \\
v(t)
\end{array}\right]+\left[\begin{array}{c}
f_{q_{a}} \\
0
\end{array}\right]} \\
& {\left[\begin{array}{c}
y_{\delta}(t) \\
y_{a}(t)
\end{array}\right]=\left[\begin{array}{cc}
0 & 1 \\
\mathcal{C}_{q_{a}} & 0
\end{array}\right]\left[\begin{array}{c}
\xi_{a}(t) \\
\delta(t)
\end{array}\right]+\left[\begin{array}{c}
0 \\
g_{q_{a}}
\end{array}\right]}
\end{aligned}
$$

with $v(t)=\bar{v}=1 \forall t \geq 0$, and the extended domain of a discrete mode $q_{a} \in Q$ is modified as

$$
\operatorname{Dom}_{a, q_{a}}^{e}=\mathbb{R}^{+} \times \operatorname{Dom}_{a, q_{a}} \times\{1\} \cup\left[0, \bar{\delta}_{q_{a}}\right] \times \mathbb{R}^{p \times m} \times\{1\}
$$

so as to impose the DT constraint.

Within this extended framework, mode $i \in Q$ is active as long as $\left(\left[\begin{array}{ll}y_{\delta} & y_{a}^{\prime}\end{array}\right]^{\prime},\left[\begin{array}{ll}u^{\prime} & v\end{array}\right]^{\prime}\right)$ keeps evolving within $\operatorname{Dom}_{a, i}^{e}$, and a transition to mode $j \neq i \in Q$ occurs as soon as $\left(\left[\begin{array}{ll}y_{\delta} & y_{a}^{\prime}\end{array}\right]^{\prime},\left[\begin{array}{ll}u^{\prime} & v\end{array}\right]^{\prime}\right)$ exits $\operatorname{Dom}_{a, i}^{e}$, and enters into $\operatorname{Dom}_{a, j}^{e}$. The reset map $\delta(t)=0$ needs to be added as soon as a mode transition occurs at time $t^{-}$.

Note that the augmented dynamics within each mode is still affine. However, the resulting dynamic matrix is not Hurwitz due to the presence of the clock. Yet, under Assumption 1, the procedure in Section 3 for model order reduction can be still adopted, in that it can be applied to the original SA system. The clock dynamics and its reset can be considered separately, and only affect the mode transitions of the reduced system via the extended domains definition.

As a consequence to the introduction of the DT, dynamics that decay in a time scale that is larger than the DT itself will be unlikely to be removed when selecting the model order through the proposed randomized approach: This is because of their contribution at the switching times when the state is reset. Finally, the length $\tau$ of the time horizon in $\mathrm{OG}_{\tau}$ and $\mathrm{COG}_{\tau}$ can be tailored to the DT value.

\subsection{Numerical example: the multi-room heating system}

We consider the example of the multi-room heating system in Section 6 and introduce a DT to the switching policy. This means that, we require that the time elapsing between two subsequent switches (heater activated/deactivated and heater turned on/off when active) must be greater than or equal to the DT. We thus increase the state vector with a clock $\delta(t)$ with dynamics $\dot{\delta}=1$, that is reset to 0 whenever a switch occurs.

Note that since the unstable dynamics of the clock do not affect the dynamics of the remaining state variables, one can apply BT to the original system without the clock. Therefore, even if the (augmented) continuous state variable of the multi-room heating system has dimension 9 , the reduction must be performed only on the original state of dimension 8 as in the example of Section 6 . The results obtained when the neglected dynamics has

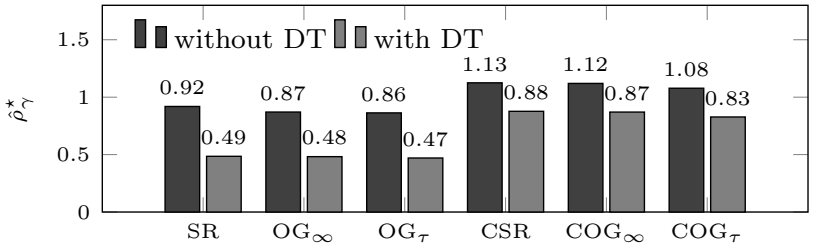

Figure 2. Quality of the reduced order model when a DT is introduced in the control policy (light bars) and when it is not adopted (dark bars).

order 1 are reported in Figure 2. The value of the optimal directional Hausdorff distance $\hat{\rho}_{\gamma}^{\star}$ is computed through Algorithm 1 for the different reset maps, but just for a value of $\gamma$ corresponding to an unitary order reduction of the asymptotically stable part of the system. The same parameter values of Section 6 are here adopted. The DT is set equal to 5 minutes (which is also the settling time of the continuous dynamics) and the time horizon length $\tau$ in the $\mathrm{OG}_{\tau}$ reset map is set equal to the DT. The $\mathrm{OG}_{\tau}$ map gives the best performance in terms of ISE with respect to the other reset maps (see Figure 2). Indeed, the DT is long enough to let the ZIR of the asymptotically stable continuous component vanish.

Not surprisingly, a comparative analysis with the values of the directional Hausdorff distance obtained without the adoption of the DT in the switching policy (see Figure 2) reveals that the quality of the reduced order model deteriorates when the DT is present, and this occurs irrespectively of the adopted reset map.

\section{Conclusions}

In this work, we proposed to extend BT to the model reduction of SA systems with endogenous switching. This involved introducing appropriate state reset maps and integrating the reduced order model design with a randomized procedure for model order selection. A comparative analysis of different maps, possibly preserving the output continuity, was performed on a benchmark example of a multi-room heating system. The approach was extended to the case of switched affine systems with DT. The proposed order selection is based on the discrepancy between the real and approximated output trajectories. If the obtained discrepancy is zero, then the reduced order model exactly reproduces the input-output behavior of the system, and it is possibly a minimal realization. A rigorous approach to exact model reduction for piecewise-affine hybrid systems is proposed in [26]. The considered class of switched systems is characterized by an endogenous switching signal. However, our method can be applied also to the case when transitions are determined by some exogenous switching signal, possibly probabilistic as in the case of Markov jump linear systems. 


\section{References}

[1] A. Abate, S. Amin, M. Prandini, J. Lygeros, and S. S. Sastry. Computational approaches to reachability analysis of stochastic hybrid systems. In Hybrid Systems: Computation and Control, volume 4416, pages 4-17. 2007.

[2] A. Abate, J. P. Katoen, J. Lygeros, and M. Prandini. Approximate model checking of stochastic hybrid systems. European Journal of Control, 16(6):624-641, 2010.

[3] A. Abate and M. Prandini. Approximate abstractions of stochastic systems: a randomized method. In 50th IEEE Conf. on Decision and Control and European Control Conf., pages 4861-4866, 2011.

[4] A. C. Antoulas. Approximation of large-scale dynamical systems, volume 6. Society for Industrial Mathematics, 2005.

[5] M. C. Campi and S. Garatti. A sampling-and-discarding approach to chance-constrained optimization: Feasibility and optimality. Journal of Optimization Theory and Applications, 148(2):257-280, 2011.

[6] M. C. Campi, S. Garatti, and M. Prandini. The scenario approach for systems and control design. Annual Reviews in Control, 33(2):149-157, 2009.

[7] A. Fehnker and F. Ivancic. Benchmarks for hybrid systems verification. In Hybrid Systems: Computation and Control, volume 2993, pages 326-341. 2004.

[8] G. Frehse. PHAVer: Algorithmic verification of hybrid systems past hytech. In Hybrid Systems: Computation and Control, volume 3414, pages 258-273. 2005.

[9] S. Garatti and M. Prandini. A simulation-based approach to the approximation of stochastic hybrid systems. In Analysis and Design of Hybrid Systems, pages 406-411, 2012.

[10] T. Geyer, F. D. Torrisi, and M. Morari. Optimal complexity reduction of polyhedral piecewise affine systems. Automatica, 44(7):1728-1740, 2008.

[11] A. Girard and C. Guernic. Zonotope/hyperplane intersection for hybrid systems reachability analysis. In 11th international workshop on Hybrid Systems: Computation and Control, pages 215-228, 2008.

[12] A. Girard, A. Julius, and G. J. Pappas. Approximate simulation relations for hybrid systems. Discrete Event Dynamic Systems, 18(2):163-179, 2008.

[13] A. Girard and G. J. Pappas. Approximation metrics for discrete and continuous systems. IEEE Trans. on Automatic Control, 52(5):782-798, 2007.

[14] A. Girard, G. Pola, and P. Tabuada. Approximately bisimilar symbolic models for incrementally stable switched systems. IEEE Trans. on Automatic Control, 55(1):116-126, 2010.

[15] J. P. Hespanha and A. S. Morse. Stability of switched systems with average dwell-time. In 38th IEEE Conf. on Decision and Control, volume 3, pages 2655-2660, 1999.

[16] J. Hu, J. Lygeros, and S. S. Sastry. Towards a theory of stochastic hybrid systems. Lecture Notes in Computer Science LNCS, 1790:160-173, 2000.

[17] A. A. Julius and G. J. Pappas. Approximations of stochastic hybrid systems. IEEE Trans. on Automatic Control, 54(6):1193-1203, 2009.

[18] X. Koutsoukos. Optimal control of stochastic hybrid systems based on locally consistent Markov Decision Processes. In Proceedings of the 2005 IEEE International Symposium on Intelligent Control, pages 435-440, June 2005.
[19] A. B. Kurzhanski and P. Varaiya. Ellipsoidal techniques for hybrid dynamics: the reachability problem. In New Directions and Applications in Control Theory, volume 321, pages 193205. 2005.

[20] D. Liberzon. Switching in systems and control. Birkhäuser Boston, 2003.

[21] J. Lunze and F. Lamnabhi-Lagarrigue, editors. Handbook of Hybrid Systems Control - Theory, Tools, Applications. Cambridge University Press, 2009.

[22] J. Lygeros and M. Prandini. Stochastic hybrid systems: a powerful framework for complex, large scale applications. European Journal of Control, 16(6):583-594, 2010.

[23] E. Mazzi, A. Sangiovanni Vincentelli, A. Balluchi, and A. Bicchi. Hybrid system reduction. In 47th IEEE Conf. on Decision and Control, pages 227-232, 2008.

[24] A. V. Papadopoulos and M. Prandini. Model reduction of switched affine systems: A method based on balanced truncation and randomized optimization. In 17th Int. Conf. on Hybrid Systems: Computation and Control, pages 113122, 2014.

[25] M. Petreczky. Realization theory of linear hybrid systems. In Hybrid Dynamical Systems, volume 457, pages 59-101. 2015.

[26] M. Petreczky and J. H. van Schuppen. Observability reduction of piecewise-affine hybrid systems. In 19th Int. Symposium on Mathematical Theory of Networks and Systems, pages 203-210, 2010.

[27] M. Petreczky, R. Wisniewski, and J. Leth. Balanced truncation for linear switched systems. Nonlinear Analysis: Hybrid Systems, 10:4-20, 2013.

[28] M. Prandini, S. Garatti, and R. Vignali. Performance assessment and design of abstracted models for stochastic hybrid systems through a randomized approach. Automatica, 50(11):2852-2860, 2014.

[29] H. R. Shaker and R. Wisniewski. Model reduction of switched systems based on switching generalized gramians. Int. Journal of Innovative Computing, Information and Control, 8(7(B)):5025-5044, 2012.

[30] C. J. Tomlin, J. Lygeros, and S. S. Sastry. A game theoretic approach to controller design for hybrid systems. Proc. of the IEEE, 88(7):949-970, 2000.

[31] C. J. Tomlin, I. Mitchell, A. M. Bayen, and M. Oishi. Computational techniques for the verification of hybrid systems. Proc. of the IEEE, 91(7):986-1001, 2003. 